\title{
ESTRATÉGIA DE CAPACITAÇÃO EM SAÚDE: UMA EXPERIÊNCIA EXITOSA DE INTEGRAÇÃO ENTRE CENTROS UNIVERSITÁRIOS
}

\section{HEALTH TRAINING STRATEGY: A SUCCESSFUL INTEGRATION EXPERIENCE BETWEEN UNIVERSITY CENTERS}

\author{
Lucrécia Helena Loureiro ${ }^{1}$, Laís Gama Dias ${ }^{2}$, Ana Cristina Souza Braga ${ }^{2}$, Maria de \\ Fátima Rocha Pinto ${ }^{1}$ \\ 1 UniFOA/ Mestrado/ lucreciahelena@gmail.com \\ 2 UBM/Enfermagem/lais.sms@epdvr.com.br \\ 2 UBM/Enfermagem/ anacris.bragaa@gmail.com \\ 1 UniFOA/Enfermagem/mrfpinto@yahoo.com.br
}

\begin{abstract}
RESUMO
O estudo buscou relatar uma experiência de capacitação de Agentes Comunitários de Saúde (ACS) realizada em conjunto entre dois centros universitários e a Secretaria Municipal de Saúde. O processo que envolve a integração ensino-serviço com um trabalho coletivo e pactuado entre docentes e discentes dos cursos de formação na área da saúde, interfere significativamente no contexto dos processos de mudança na formação dos profissionais de saúde. Com a finalidade de contribuir neste processo de mudança, optamos por iniciar a capacitação com os Agente Comunitário de Saúde. Com objetivo de preparar os ACS para o desenvolvimento de ações de promoção, prevenção e atenção e principalmente analisar e avaliar o impacto que a capacitação realizada contribuiu no dia a dia das atividades desenvolvidas pelas equipes da Estratégia Saúde da Família (ESF). As oficinas aconteceram entre setembro e dezembro de 2015, com 179 ACS, destes, 76,5\% apresentaram aproveitamento na Capacitação, sendo 80,4\% do sexo feminino. $\mathrm{Na}$ análise e sistematização dos dados e informações, foi possível identificar e caracterizar a percepção dos profissionais gerentes que atuam nas ESF sobre a compreensão da educação permanente e a avaliação das iniciativas implementadas no processo de trabalho. Conclui-se que a integração entre as Universidades e o serviço é um ponto chave na Gestão do Trabalho.
\end{abstract}

Palavras-chave: Estratégia Saúde da Família, Agente Comunitário de Saúde, Processo de Trabalho, Ensino

\begin{abstract}
The study sought to report a training experience of Community Health Agents (ACS) carried out jointly between two university centers and the Municipal Health Department. The process involving the teaching-service integration with a collective work and agreed between teachers and students of the training courses in the health area, significantly interferes in the context of the processes of change in the training of health professionals. In order to contribute to this process of change, we opted to start the training with the Community Health Agent. In order to prepare the ACS for the development of actions of promotion, prevention and attention and mainly to analyze and evaluate the impact that the training carried out contributed in the day to day activities developed by the Family Health Strategy (ESF) teams. The workshops took place between September and December 2015, with 179 ACS, of which, 76.5\% were


proficient in the Training, with $80.4 \%$ being female. In the analysis and systematization of the data and information, it was possible to identify and characterize the perception of the professionals professionals who work in the FHT on the understanding of the permanent education and the evaluation of the initiatives implemented in the work process. It is concluded that the integration between the Universities and the service is a key point in the Work Management

Keywords: Family Health Strategy, Community Health Agent, Work Process, Education.

\section{INTRODUÇÃO}

Com o advento da globalização e a consequente competitividade no mercado de trabalho, a exigência por profissionais capacitados está cada vez maior. Neste campo da competitividade, não é necessário somente o domínio da técnica, mas um saber amplo, integralizado e critico.

$\mathrm{Na}$ Área da Saúde, várias discussões trazem à tona estas questões. A capacitação de profissionais da área de saúde requer a adoção de Estratégias de ensino que estejam em consonância com a política de saúde vigente no país de um lado e do outro o compromisso de garantir os direitos dos cidadãos que necessitam de atendimento ao nível da atenção primária.

Em seu estudo sobre potencialidades da Educação Permanente para a transformação das práticas de saúde, Lopes,et.al (2007), demarcaram o momento em que a estratégia da educação permanente passou de programa de formação para uma Política Nacional.

Outro detalhe importante relativo à Educação Permanente e que é bem ressaltado por Pierantini et al.( 2008) ,é que o Brasil tem desenvolvido múltiplas iniciativas no sentido de produzir conhecimento que possa contribuir para o fortalecimento de ações na área da gestão do trabalho.

Dentro desta ótica, Andrade ( 2015), aponta que em nível federal, o marco para a integração entre ensino e serviço se deu com a criação da Comissão Interministerial de Gestão do Trabalho e da Educação em Saúde, vinculada aos Ministérios da Educação e da Saúde.

Essa situação reflete a mesma encontrada por Nideck (2015) quando afirma que “contemplar a interface educação e trabalho na perspectiva deleuze--guattariana 
significa resistir ao empreendimento de educação para a empregabilidade e reconhecer o ato de aprender como tarefa infinita”.

Baseadas em preocupações principalmente com os profissionais recémcontratados especificamente os Agentes Comunitários de Saúde - ACS, um personagem muito importante na implementação do Sistema Único de Saúde, fortalecendo a integração entre os serviços da Atenção Primária e a comunidade.

Estes profissionais necessitam de uma formação mais voltada a atender as demandas do Sistema de Saúde e da população, e o que estimula a realização de capacitação que contemplem o desenvolvimento de atividades que articulem o ensino, a pesquisa e o trabalho para o cuidado em saúde, com base nas necessidades sociais e considerando a capacidade de promover o desenvolvimento no enfrentamento de problemas de saúde prevalentes dentro da realidade do serviço.

A inserção do agente comunitário de saúde (ACS) na rede do Sistema Único de Saúde (SUS) tem suscitado polêmica a respeito da forma mais adequada de relação de trabalho que deve ser adotada para que se possa contar de modo sustentável com esse tipo de recurso humano (NOGUEIRA,2000).

Reconhecidamente o trabalho do agente comunitário de saúde lida com demandas relativas às condições de vida, à política, à cidadania e à organização dos grupos e suas relações, incluindo a família. Desta forma, urge o conhecimento do campo da saúde, além dos saberes que perpassam o cotidiano da sua atuação prática e esse saber precisa estar sistematizados na formação dos ACS. Para a realização de um trabalho de qualidade no campo da saúde, independentemente da realidade local que esses profissionais atuam.

Na década de 80, iniciou-se um clima de reestruturação do modelo de saúde vigente, e esta reforma do modelo de saúde ocorreu com a criação do Sistema Único de Saúde (SUS). Com o novo modelo, onde as ações de saúde na lógica organizativa dos serviços, com universalidade, integralidade, como diretrizes básicas da porta de entrada do sistema, priorizam as ações preventivas

em detrimento as curativas.

Em 1994, o Ministério da Saúde, decidiu reorganizar o modelo de atenção a saúde vigente nos pais e instituiu o Programa de Saúde da Família. A grande maioria dos municípios aderiu ao programa e iniciaram com a nova política de atendimento a população. Para a implantação do programa, foi necessária a contratação de uma equipe 
multidisciplinar, composta de médico, enfermeiro, técnicos de enfermagem e Agentes Comunitários de Saúde, em busca de uma efetivação e sofisticação da Atenção Primária a Saúde.

Com um novo olhar para a saúde da população o foco passou a ser a Saúde e não a doença, a família e não o individuo. A comunidade passou a ser assistida por uma equipe e não somente por um profissional, desta forma a ESF, propõe à melhoria da qualidade de vida dessas pessoas.

A ESF é um modelo assistencial que prioriza as ações de saúde em um contexto multiprofissional na atenção básica onde uma equipe formada por profissionais de diferentes áreas ajuda a enfrentar com qualidade os muitos determinantes sociais de saúde. Hoje se privilegia o trabalho interdisciplinar em detrimento ao individualismo das ações, não existe mais o foco em somente uma área do conhecimento capaz de dar respostas à complexidade humana, faz necessária a intervenção multiprofissional, haja vista as tantas desigualdades sociais. Acredita-se que os saberes se estabelecem com as trocas.

Uma organização ao contratar um funcionário, espera que esta pessoa consiga atender suas expectativas, atingir ou superar metas. Para que isso ocorra à organização disponibiliza de educação permanente para o seu quadro efetivo.

Constata-se a necessidade de uma maior valorização da Educação Permanente para a Gestão do Trabalho e Educação em Saúde. É de salutar importância solicitar parcerias com os estágios curriculares de enfermagem na rede SUS, visando demarcar as ações da educação permanente do Município e contribuir para a definição de uma política de estágios com maior integração ensino-serviço.

\section{MATERIAIS E MÉTODOS}

Trata-se de um estudo de caso, descritivo e observacional, com uma estratégia de capacitação dos ACS em parceria entre a Secretaria Municipal de Saúde e os dois centros universitários da região, representados pelos docentes e discentes do curso de Graduação em Enfermagem. A tática foi desenvolvida no Município de Volta Redonda, no período de setembro a dezembro de 2015. 
O Município também conhecida como a "Cidade do Aço", está localizado na microrregião do Vale do Paraíba, na mesorregião Sul Fluminense no estado do Rio de Janeiro, no Brasil. Com uma população estimada em 2015 de 262.970 habitantes, o que torna a maior cidade da região Sul Fluminense e a terceira maior do interior do estado.

A cidade possui uma das redes de saúde mais completas do interior do estado, sendo, no entanto, referência para toda região do Vale do Paraíba Fluminense, contando com 43 unidades de saúde, sendo 35 ESF (17 no distrito sanitário Norte e 18 no Sul) e 08 unidades básicas.

O município está dividido em dois grandes territórios: o Distrito Sanitário Norte (na margem esquerda do Rio Paraíba do Sul) e o Distrito Sanitário Sul (na margem direita).

O curso foi organizado pela Superintendência de Gestão do Trabalho e Educação em Saude- SGTES, Superintendência de Atenção e Vigilância em Saúde- SAVS em parceria com os Centros Universitários de Volta Redonda UniFOA e Barra Mansa-UBM.

Primeiramente após o surgimento da proposta de capacitação dos Agentes Comunitários de Saúde em imunização, leitura e acompanhamento do cartão vacinal das crianças, o projeto foi escrito e apresentado em reunião de superintendência e núcleo de gestão, e aprovado sua execução pela Secretária de Saúde.

Em seguida os Centros Universitários da região foram contatados para estabelecer parceria, e uma nova reunião aconteceu com a presença de representantes legais de ambas as Instituições de Ensino Superior- IES; na ocasião o SGTES entregou a proposta do projeto, os mesmos apoiaram a minuta e levaram para discussão junto ao corpo docente.

Posteriormente, o SGTES recebeu um e-mail das IES com o aceite da proposta, aprestando a designação das docentes responsáveis pelo projeto colocando-se a disposição da SMS para desenvolver o trabalho proposto.

Com a perspectiva de iniciar a capacitação após o recesso das atividades acadêmicas, estabeleceu-se uma data para encontro entre as IES. Em ocasião as responsáveis em conduzir o projeto, sugeriram uma readaptação no projeto proposto pela SMS, com inclusão de novas discussões a respeito do assunto a ser 
abordado. As propostas foram analisadas e apresentadas as Superintendências e aprovadas por todas as instâncias colegiadas dentro da secretaria.

Paralelamente, a Coordenação dos Distritos/SAVS, iniciou a articulação e confecção de escalas de revezamento para viabilizar a ausência dos funcionários no período do curso e a entrega de vale transporte para deslocamento.

A população alvo deste estudo foi constituída pelos ACS do Município de Volta Redonda. Esses funcionários são recém contratados através de um processo seletivo realizado em 2015 para atuarem na Estratégia Saúde da Família. Para identificação de ACS por distrito sanitário (norte e sul), procedeu-se a distribuição dos mesmos por oficinas. Após essa divisão, o registro de funcionários totalizou 179 colaboradores, que serviu de base para a obtenção da amostra.

Foram $16 \mathrm{~h}$ de treinamento para cada participante, perfazendo um total de 04 encontros de 4horas. Os encontros aconteceram uma vez por semana, as segundas feiras, com duas turmas, nos horários de $8 \mathrm{~h}$ às $12 \mathrm{~h}$ e de $13 \mathrm{~h}$ as $17 \mathrm{~h}$, um grupo em local cedido por uma das Universidades envolvidas e o outro no espaço de Educação em Saúde do Município, localizado no estádio da cidadania.

Todos os participantes receberam uma apostila elaborada pelas IES e viabilizada pela SGTES, no primeiro dia do treinamento, com todo o conteúdo programático. Utilizaram-se como estratégia de ensino, vídeos motivacionais, slides, dinâmicas e a utilização do Data Show.

Ao final do treinamento, decidimos publicar um artigo, relatando essa experiência. Para realização do estudo solicitamos, inicialmente, a permissão da Secretaria Municipal de Saúde e após sua concordância iniciamos uma pesquisa em toda a documentação entregue pelas monitoras, destacamos as listas de presença e a avaliação do curso realizada pelas as enfermeiras gerentes das unidades capacitadas.

\section{RESULTADOS E DISCUSSÃO}

Na ESF, a promoção à saúde implica um novo olhar dos profissionais ACS, vislumbrando um conceito ampliado de saúde, tendo neste contexto a Imunização como pano de fundo. Nesse sentido, para suprir as lacunas de formação destes 
profissionais para esse novo olhar, o processo de educação permanente em saúde é essencial.

Nesse Município do Interior do Estado do Rio de Janeiro, que foi cenário de nossa pesquisa, o processo de educação permanente está presente no cotidiano da ESF, uma vez que nele se desenvolve um projeto de articulação teoria e prática, onde as Universidades da Região, em parceria com a Secretaria Municipal de Saúde, foram convidadas a promover a capacitação dos ACS em Imunização, com o intuito de qualificar esses profissionais.

Os Enfermeiros Gerentes desse Município, contribuíram para avaliar o curso de Imunização para ACS, ministrados pelos docentes e discentes do curso de graduação em enfermagem. Esses gestores referem que o processo de educação permanente traz contribuições para a qualificação dos ACS, para a identificação de possíveis falhas nas orientações na leitura do cartão vacinal, para a conscientização das doenças que acometem a população e suas necessidades reais de saúde, na organização e funcionamento do serviço, para o trabalho em equipe, aperfeiçoamento, atualização e mudanças na prática.

Os dados da avaliação foram sistematizados e permitiram caracterizar a percepção dos profissionais gerentes que atuam nas equipes saúde da família sobre a compreensão da educação permanente e a avaliação das iniciativas implementadas no processo de trabalho.

Para atingir o objetivo pretendido realizou-se um projeto, denominado, "Integração Ensino- Serviço na formação do agente comunitário de saúde", como mostra o organograma ilustrativo abaixo com as principais fases: 
Projeto: Integração Ensino- Serviço na formação do agente comunitário de saúde

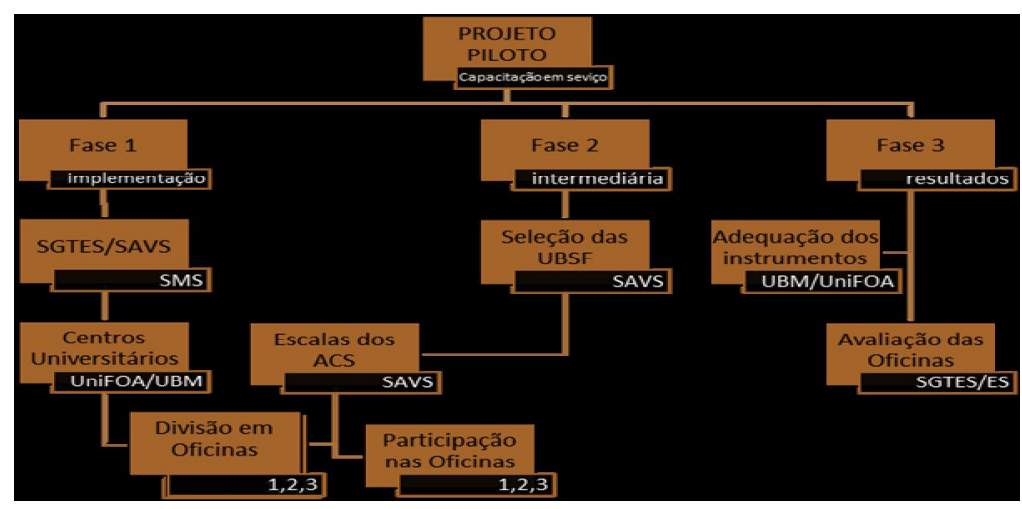

O organograma exemplifica as etapas do processo de construção do projeto. Na fase dos resultados avaliamos que a maioria $(80,4 \%)$ dos ACS que fizeram parte da pesquisa e que atuam nas equipes é do sexo feminino, sendo o predomínio de funcionários atuando no Distrito Sanitário Sul (53,0\%). Importante frisar que os dados referentes ao gênero dos profissionais, confirmam a presença da feminização na força de trabalho existente na área da saude (gráfico 1).

Gráfico 1. Avaliação por gênero

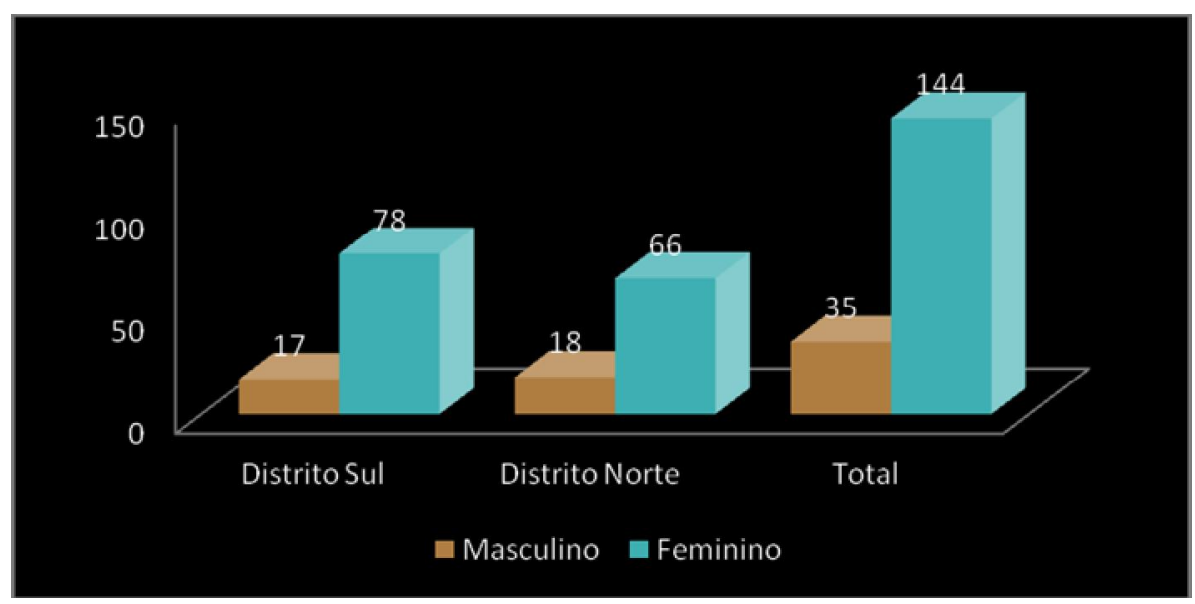

Ao passo que das Unidades de ESF existentes no município somente (46,2\%) foram contempladas com a capacitação, conforme gráfico 2. 


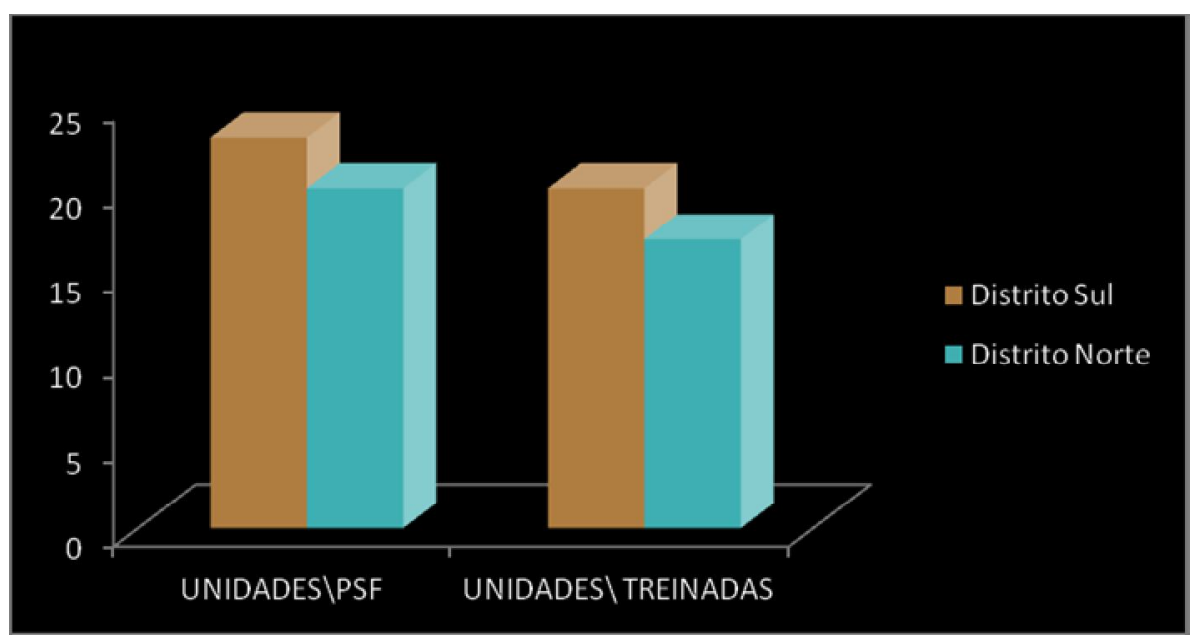

A partir da análise das listas de presença, constata-se um dado relevante que $(76,5 \%)$ dos profissionais apresentaram aproveitamento na Capacitação conforme demonstrado no grafico 3 .

Grafico 3. Avaliaçao presencial por Distrito Sanitário

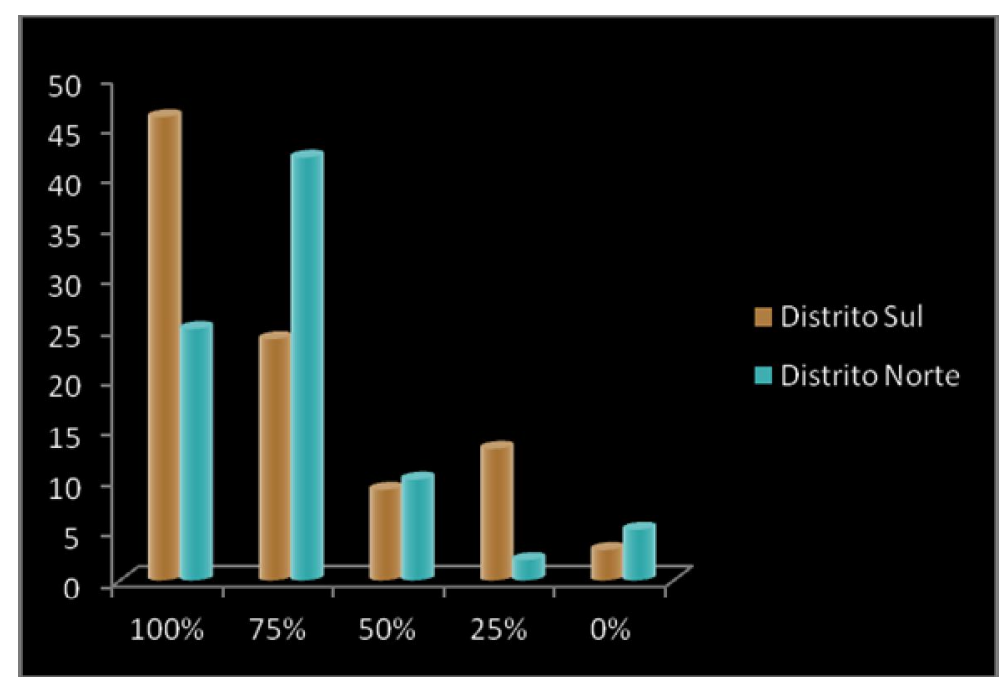

No gráfico 4 são apresentados os dados relativos ao impacto da capacitação no processo de trabalho desenvolvido pelos ACS.

Foi inquirido aos gerentes se o curso realizado contribuiu para a melhoria no processo de trabalho. 
Gráfico 4. Impacto das avaliações, 2015

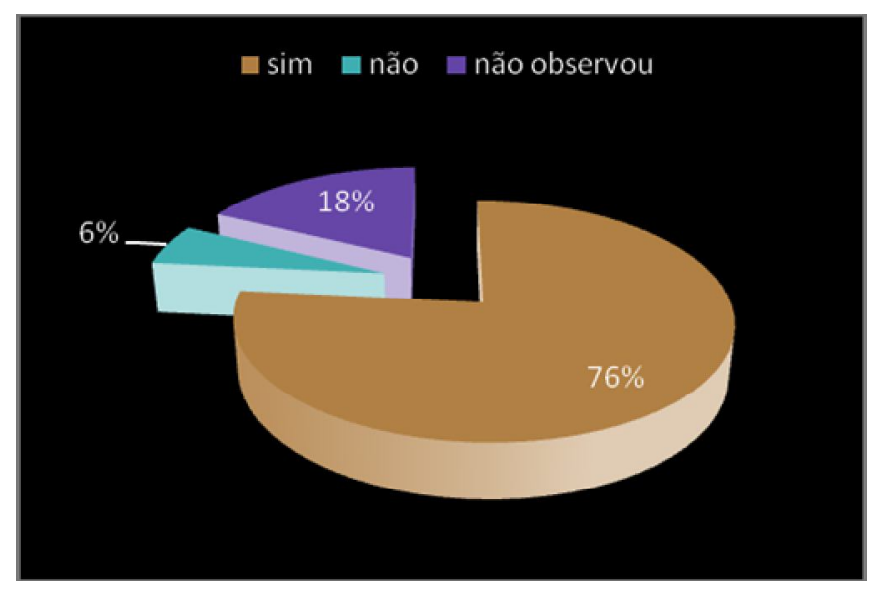

Fonte: Avaliação do curso de ACS

Nas avaliações dos Gerentes (G), somente 39,5\% responderam a pesquisa, destes $76,4 \%$ mostram que a capacitação em imunização realizada em parceria com as universidades, contribuiu para a qualificação dos trabalhadores, reforçando o aprender a trabalhar com a comunidade, principalmente na identificação do esquema vacinal:

"Foi interessante, pelo menos os ACS estão se esforçando para entender e interpretar o cartão de vacina. Eles olham junto com os técnicos e também conosco os enfermeiros". (G 3)

"Comentaram que ficaram mais seguros para orientação sobre o tema".(G12; G6)

"Eles entenderam o esquema, se interagiram da problemática do assunto, muito longo e também muito interessante”. (G 9; 1)

"Foi muito bom e vai nos ajudar a melhorar nosso processo de trabalho". (G 10; 6)

"Capacitação muito produtiva, contribuindo bastante para o trabalho do dia a dia. Cooperando para melhor orientação da Comunidade".(G 11)

Os aspectos evidenciados nessas falas mostram que a parceria estabelecida entre as instituições de ensino e a SMS, possibilitou um aprendizado mutuo, à integração, ao trabalho em equipe e à responsabilidade com a comunidade.

A educação deve servir para preencher lacunas do conhecimento, para identificar os possíveis problemas no cotidiano de trabalho e com isso contribui ISSN 1983-7011 
Ensino, Saúde e Ambiente -V10 (3), pp. 34-47, Dez. 2017

para a transformação das práticas profissionais e da organização do trabalho.(MS,2005).

A integração da equipe auxilia no processo de aprendizado. O trabalho em equipe é uma tendência atual, uma vez que, frente à complexidade e diversidade dos problemas vivenciados na prática, os profissionais devem desenvolver competências associadas à capacidade de cooperação, na resolução de problemas.(SCHIMITH， 2009; SILVA,2010;SOUZA,2010; OLIVEIRA,2011; PAULINO et.al, 2012).

Ao passo que $23.5 \%$ dos enfermeiros gerentes que responderam a avaliação revelam que as equipes de $\mathrm{ACS}$, onde eles trabalham, até o momento não demonstraram modificação no processo de trabalho devido a inúmeras atividades priorizadas nos meses subsequentes ao curso que impediram o desenvolvimento das competências e habilidades adquiridas durante a capacitação:

"Ainda não observei o impacto da capacitação no processo de trabalho, devido a inúmeras outras atividades desenvolvidas que foram priorizadas". (G 14)

"O conteúdo do curso ainda não contribuiu com o processo de trabalho".(G 17; $16 ; 15)$

\section{CONSIDERAÇÕES FINAIS}

No que se refere à qualificação do ACS, apesar do Ministério da Saúde definir as principais diretrizes para sua formação, na prática esse processo ainda é muito diversificado. Acreditamos que na qualificação reside a força e a riqueza da presença desse ator em uma equipe de saúde.

A qualificação dos trabalhadores permite uma nova visão do processo de trabalho, a necessidade de capacitação dos Agentes Comunitários de Saúde, que estão iniciando sua vida profissional em uma atividade, simples e ao mesmo tempo complexa. O ACS, querendo ou não participa e circula dentro de uma comunidade, das casas, convivem com o cotidiano da comunidade o qual faz parte.

No Município, a lógica em relação à satisfação do profissional também sofreu alteração, a capacitação levou os profissionais a se preocuparem mais em organizar o trabalho de forma a dar aos usuários mais satisfação quando acessam os serviços oferecidos. Cada diretriz do treinamento foi estudada para que o maior 
número de informações possíveis fosse transmitido à equipe de ACS e apontamos que os dois períodos tiveram avaliações estatisticamente similares.

Por fim, valer notar que as expectativas depositadas em torno da participação dos centro universitários foi de fundamental importância e atingiu todas as expectativas da SGTES, que aposta que esta parceria poderá permanecer nos próximos anos, atingindo outros atores envolvidos no processo de trabalho dentro da Secretaria Municipal de Saúde.

\section{REFERÊNCIAS}

ANDRADE, S. R. de; BOEHS, Astrid Eggert and BOEHS, Carlos Gabriel Eggert. Percepções de enfermeiros docentes e assistenciais sobre a parceria ensinoserviço em unidades básicas de saúde. Interface (Botucatu) [online]. 2015, vol.19, n.54, pp. 537-547. ISSN 1807-5762.

BERNARDO, V. Desenvolvimento de programa multimídia aplicado ao ensino da medicina. Tese de Mestrado, EPM/UNIFESP, 1996.

BRASIL. Ministério da Saúde. Programa de Saúde da Família. Brasília, COSAC,1994.

BRASIL. Constituição Federal de 1988. Brasília: SENADO FEDERAL, 1988. . Lei 8080 de 19 de setembro de 1990. . Lei 8142 de 28 de setembro de 1990.

LOUREIRO, L.H. ENSINO DE GERÊNCIA EM SAÚDE COLETIVA E A EDUCAÇÃO PERMANENTE DOS PROFISSIONAIS DA ESF : o uso do software educativo / Lucrecia Helena Loureiro. - Volta Redonda:UniFOA, 2010.68 f.Dissertação (Mestrado Profissional) -- Centro Universitário de Volta Redonda -UniFOA. Pós-graduação em Ensino em Ciências da Saúde e do Meio Ambiente, 2010 .

LOTTA, g. s. saber e poder: agentes comunitários de saúde aproximando saberes locais e políticas públicas / Gabriela Spanghero Lotta. 2006. orientador: Peter k. Spink.dissertação (mestrado) - escola de administração de empresas de são paulo.

MARTINES, W.R; CHAVES,E.C. Vulnerabilidade e sofrimento no trabalho do Agente Comunitário de Saúde no Programa de Saúde da Família. Rev Esc Enferm USP. 2007; 41(3):426-33. 
MINISTÉRIO DA SAÚDE (BR). Secretaria de gestão do trabalho e da educação na saúde. documentos preparatórios para 3 a conferência nacional de gestão do trabalho e da educação na saúde. Brasília (DF): Ministério da Saúde; 2005.

NIDECK, Renata de Lima Pacheco and QUEIROZ, Paulo Pires de. Perspectivas para o ensino na saúde: do 'apagão educacional' à política de educação permanente. Trab. educ. saúde [online]. 2015, vol.13, n.1, pp. 159-180. Epub Dec 02, 2014. ISSN 1981-7746.

NOGUEIRA, R., RAMOS, Z.V.O. A vinculação institucional de um trabalhador sui generis - o agente comunitário de saúde. [Texto para discussão 735]. 2000. 33p. Disponível em <URL: http://www.ipea.gov.br >. Acesso em: jun 2012.

NUNES, M.O; TRAD,B.L;ALMEIDA,,B.A; HOMEM,C.R;MELO,M.C. AGENTE COMUNITÁRIO DE SAÚDE: personagem híbrido e polifônico. Cad. Saúde Pública, Rio de Janeiro, 18(6):1639-1646, nov-dez, 2002.

OLIVEIRA W.M.A; BEZERRA A.L.Q. Autoavaliação da estratégia saúde da família por enfermeiros. Rev enferm UERJ. 2011; 19:20-5

PAULINO V.C.P, BEZERRA A.L.Q, BRANQUINHO N.C.S.S, PARANAGUÁ T.T.B. Educação permanente e saúde da família. rev. enferm. uerj, rio de janeiro, $2012 \mathrm{jul} / \mathrm{set} ; 20(3): 312-6$.

PIERANTONI, C.R. Trabalho e Educação em Saúde no Mercosul. Brasília, DF: Ministério da Saude; Rio de Janeiro: Europa, 2008.

SILVA, J.A O agente comunitário de saúde do Projeto QUALIS: agente institucional ou agente de comunidade? Dissertação (Mestrado) Faculdade de Saúde Pública, Universidade de São Paulo São Paulo, 2001. Tese (Doutorado) Faculdade de Saúde Pública, Universidade de São Paulo.

SILVA, A.S; DALMASO. A.S.W. O AGENTE COMUNITÁRIO DE SAÚDE E SUAS ATRIBUIÇÕES: os desafios para os processos de formação de recursos humanos em saúde. Interface - Comunic, Saúde, Educ, v6, n10, p.75-94, fev 2002.

SILVA L.A.A, FERRAZ F, LINO M.M, BACKES V.M.S, SCHMIDT S.M.S. Educação permanente em saúde e no trabalho de enfermagem: perspectiva de uma práxis transformadora. Rev Gaúcha Enferm. 2010; 31:557-61

SCHERER, M. D. A. et al. Rupturas e resoluções no modelo de atenção à saúde : reflexões sobre a estratégia saúde da família com base nas categorias Kuhnianas, Interface - Comunic., Saúde, Educ., v.9, n.16, p.53-66, set.2004/fev.2005.

SCHIMITH M.D, LIMA M.A.D.S. O enfermeiro na equipe de saúde da família: estudo de caso. Rev enferm UERJ. 2009; 17:252-6. 13. 
SOUZA, R. A. e CARVALHO, A. M. Programa de Saúde da Família e Qualidade de Vida: um olhar da psicologia. Revista Estudos de Psicologia, 8(3), 515-523, 2003.

SOUZA R.C.R, SOARES E, SOUZA I.A.G, OLIVEIRA J.C, SALLES R.S, CORDEIRO C.E.M. Educação permanente em enfermagem e a interface com a ouvidoria hospitalar. Rev RENE. 2010; 11(4):85-94. 18.

TEIXEIRA, C.F., PAIM, J.S. Planejamento e programação de ações intersetoriais para a promoção da saúde e da qualidade de vida. Rev. Adm. Pública, v.34, n.6, p.63-80, 2000. 